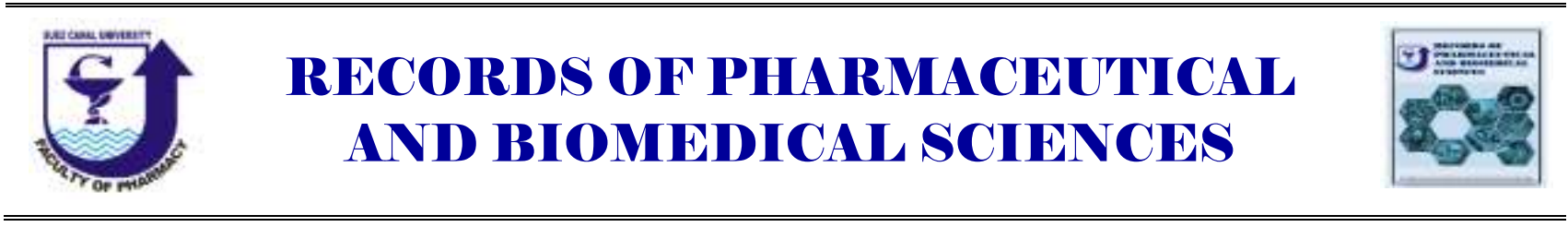

\title{
Recent insight into BACE1 as a potential target for treatment of Alzheimer's disease
}

\author{
Asmaa M Atta ${ }^{a}, \quad$ Khaled M. Darwish ${ }^{b}$, Mohamed S. Elgawish ${ }^{b}$, Samia M. Mostafa ${ }^{b}$ Mohamed $^{-}$ \\ A. Helal ${ }^{b, c}$ \\ ${ }^{a}$ pharmaceutical chemistry department, Faculty of Pharmacy, Badr University, Cairo, Egypt \\ ${ }^{b}$ Medicinal Chemistry Department, Faculty of Pharmacy, Suez Canal University, Ismailia, Egypt \\ ${ }^{c}$ Biomedical Sciences Program, University of Science and Technology, Zewail City of Science and Technology, \\ Giza, Egypt
}

\section{Received on:}

\section{Revised on:}

Accepted on:

Correspondence Author:

Tel: +201227268096

E-mail address:

Ph.asmaaatta@yahoo.com

\begin{abstract}
Alzheimer's disease (AD) is one of the most common progressive neurodegenerative diseases that represents about $60-80 \%$ of total cases of dementia all over the world. Beta secretase (BACE1) is a membrane-anchored aspartic protease which cleaves the peptide bond between Met671-Asp672 of APP (amyloid precursor protein) where its activity is the rate limiting step of producing amyloid $\beta$, the primary neuropathological hallmark of Alzheimer's disease (AD). Therefore, inhibitors of this enzyme have the potential to be a promising and attractive target for intervention of potent therapeutics for treatment AD. Actually, there are many challenges facing the development of effective drug-like BACE1 inhibitors including penetration of BBB and selectivity against other proteases. However, many efforts have been devoted by pharmaceutical industries and academia for development of small size, potent, selective and biologically active BACE1 inhibitors. Till now, none of which is FDA approved but some of which have exhibited clinical potential. In this article, we outlined overview of beta secretase inhibitors that have been designed and developed for treatment of $\mathrm{AD}$.
\end{abstract}

Keywords: Alzheimer's disease; AD; BACE1; BACE1 inhibitors.

\section{Introduction}

Alzheimer's disease (AD) is one of the most common progressive neurodegenerative diseases that represents about $60-80 \%$ of total cases of dementia all over the world (Femminella et al. 2018). According to World's Alzheimer's report 2018, there is at least 50 million people are living with AD or other dementias worldwide (Patterson 2018). Furthermore, it is expected that this number of people will reach 74.4 million by 2030 and 131.5 million by 2050 which will represent a considerable burden on health organization (Ali et al. 2015). AD is the sixth leading cause of death in the United
States and the fifth leading cause of death among Americans age 65 and older (Reports 2018). Nevertheless, till now there are only 5 drugs have been approved by food and drug administration (FDA) for treatment of $\mathrm{AD}$ including: tacrine (approved 1993 but was discontinued due to hepatotoxicity), donepezil; 1996, rivastigmine; 1998, galantamine; 2001 and memantine; 2004 (2020 Alzheimer's disease facts and figures 2020). These drugs or their combination usually relieve symptoms only without effect on progression of neurodegeneration process in $\mathrm{AD}$. Therefore, the current efforts are orientated toward evolution of 
new drugs that can target the mechanism of disease and inhibit its progressive neuronal deterioration. Pathologically, the presence of $\beta$-amyloid plaques and intraneuronal neurofibrillary tangles in the brain are the major neuropatholgical hallmarks of AD (C Vickers et al. 2016) Fig. (1). It was proposed according to amyloid hypothesis (the most accepted hypothesis illustrated the pathogenesis of AD yet) that $\beta$-amyloid peptide $(A \beta)$ is the main component of amyloid plaques. $A \beta$ is a peptide produce of amyloid precursor protein (APP) which is transmembrane protein consists of about 695 amino acid residues, highly expressed in neuron cells. APP is alternatively processed by two various pathways ; non-amyloidogenic pathway and amyloidogenic pathway (Nhan, Chiang, and Koo 2015). Under pathological conditions, APP is cleaved by $\beta$ secretase (BACE-1) releasing smaller soluble Nterminal fragment $\mathrm{sAPP} \beta$ and CTF $\beta$ (C-terminal fragment). Further cleavage of CTF $\beta$ by $\gamma$-secretase produces AICDs and $\beta$-amyloid peptide (A $\beta$ monomers) (Nhan, Chiang, and Koo 2015). There are many species of $A \beta$ where $A \beta 40$ (with 40 amino acid) and $A \beta 42$ (with42 amino acid) are the most predominant in the brain (Korczyn 2008). Regardless of their similarities, $A \beta 42$ is more susceptible to aggregation and fibrillation and considered as the most toxic $A \beta 42$ species in pathogenesis of AD (Kontush 2004). The accumulation of $\mathrm{A} \beta$ into amyloid plaques in the brain stimulates many neurotoxic processes such as increased oxidative stress, neuroinflammatory response, synaptic dysfunction, hyperphosphorylation of tau protein, apoptosis and ultimately neuronal death (Rhein and Eckert 2007). Therefore, B-secretase enzyme and its inhibition have emerged as a valuable promising target for development of treatment that may halt the progression of $\mathrm{AD}$.

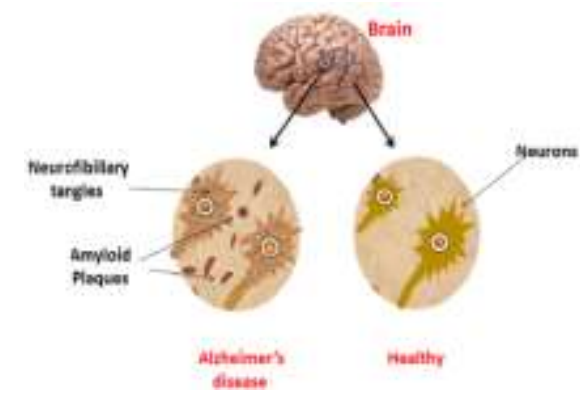

Fig. (1): Healthy brain versus Alzheimer's diseased brain

\section{Beta secretase (BACE1) as a therapeutic target for treatment of $\mathrm{AD}$}

Beta secretase (BACE1: beta site APP cleaving enzyme I) also known as memapsin 2 (Lin et al. 2000) or Asp2 (Yan et al. n.d.) is a membraneanchored aspartic protease of pepsin family. It was first discovered by various research groups in 1999 during their study of deposition of amyloid- $\beta$ in $\mathrm{AD}$ (Sinha et al. 1999). It cleaves the peptide bond between Met671-Asp672 of APP (amyloid precursor protein) where its activity is the rate limiting step of producing amyloid $\beta$ (Vassar et al. 1999), the main component of accumulated plaques that observed in brains of AD patients. In addition, it was proved that reducing BACE1 is enough to inhibit generation of amyloid- $\beta$ in neurons and brains of knockout animals (Harrison and Beher 2003). Therefore, inhibitors of this enzyme have the potential to be a promising target for intervention of AD. Recently, there are many efforts have been pursued all over the world in order to develop and find out inhibitors that can efficiently halt BACE1.

\subsection{Crystal structure of BACE1}

Generally, structure of BACE1 is like other aspartic proteases. However, it has characteristic features in the active site, which is less hydrophobic and more open than other aspartic proteases. BACE1 is a monomeric protein that highly expressed in neurons of the brain and has 501 amino acid residues. It consists of 3 major domains: N-terminal domain, transmembrane domain and $\mathrm{C}$-terminal domain (Lin et al. 2000). Bilobal structure of BACE1 shows the conserved folding of aspartic proteases where the substrate binding cleft lies between $\mathrm{N}$ - and Cterminal domains (Hong et al. 2000). The catalytic site includes two aspartic residues Asp228 and Asp32 (catalytic dyad) located in the center of the cleft. Recently, X-ray and number of theoretical studies revealed that one of Asp residues exists in protonated form while the other Asp remains unprotonated within catalysis process (Coates et al. 2006) . Active site of BACE1 is distinguished by existence of hydrophilic and hydrophobic pockets and is sheltered by flexible hairpin loop (named also flap) between Val67 and Glu77 where the key residue is Try71 (Shimizu et al. 2008) (Fig.2).

Flap is the most flexible part in the active site that located in N-terminal domain covering the cleft and is perpendicular to it like other aspartic protease (Hong and Tang 2004). Changes in flap conformation regulate the entry of substrate in active site and adjust its geometry for effective catalysis process (Shimizu et al. 2008). It has been reported that open conformation of the flap is observed in substrate free (apo) structure of BACE1 
where flap moves away from aspartic dyad with absence of hydrogen bond between flap residues Try71 and Try76; however the flap adopts a closed conformation in case of inhibitor-bound form where it moves toward catalytic aspartic dyad and a phenolic hydroxyl group of Try71 flap residue formed a hydrogen bond with $\mathrm{NH}$ of Try76 side chain (Fig.3) (Shimizu et al. 2008).

Furthermore, there is another flexible loop located near N terminus (near Ser10), known as 10s loop (Lys9 - Try14). There are two conserved water molecules were observed in active site of BACE1 similar to the other aspartic protease (Shimizu et al. 2008). The first water molecule is located between two aspartic dyad Asp 228 and Asp32 of BACE1 and suggested to help in catalysis process. While the second conserved water molecule is shared in a hydrogen bond with Try71 residue in the flap in addition to its participation in hydrogen bonding network to stabilize flap in closed conformation.

\section{Evolution of Beta-secretase inhibitors}

Evolution of effective drug-like BACE1 inhibitors has many obstacles. Initially, BACE1 inhibitors must cross blood brain barrier (BBB) and neuronal membrane to access BACE1 which located in the brain (Halima et al. 2016). Additional challenge that should be taken into consideration is the

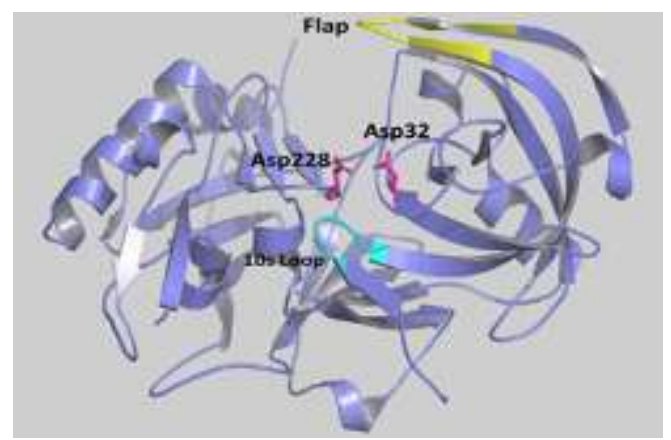

Fig (2): Crystal structures of isolated BACE1, highlighting the active site aspartate residues: Asp32 and Asp228 (red spacefill representation), hairpin loop termed 'flap' (orange) and the 10s loop (green).

characteristic structure of enzyme including: catalytic dyad, structural flexibility and large catalytic pockets (Yuan et al. 2013). Therefore, inhibitors are proposed to be in large size to interact efficiently with enzyme active site. But these large inhibitors exhibit low in vivo activity and they should be small in size to show promising drug-like absorption, distribution, metabolism and excretion (ADME) properties. Moreover, selectivity against other aspartic proteases is particularly substantial. Many off targets side effects may be occurred due to inhibition of other aspartic proteases (BACE2, pepsin, cathepsin $\mathrm{D}$, cathepsin $\mathrm{E}$ and renin) by unselective BACE1 inhibitors (A. K. Ghosh and Osswald 2014).

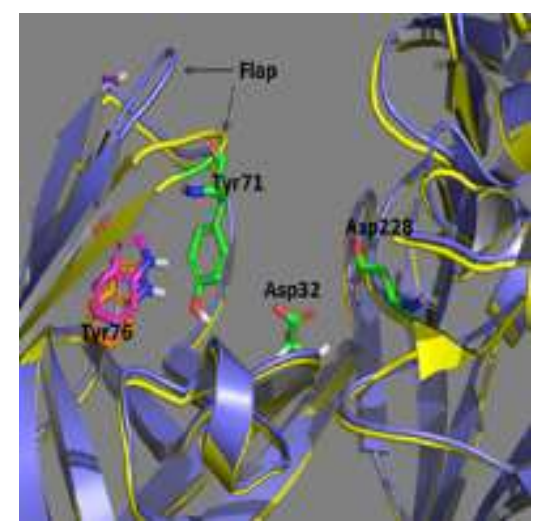

Fig. (3): Superimposed crystal structure of open flap conformation (PDB ID: 2OHU, with blue color) and close- flap conformation (PDB ID: 1W51, with yellow color) of BACE1 crystallography (ligand was deleted for clarity).

\subsection{Substrate-based (peptidic) inhibitors}

Ligandability of BACE1 was first elucidated with development of first highly potent substrates-based inhibitor OM99-2 (inhibitor 1). Design of OM99-2 (Fig. 4) was based on simulation of BACE substrate at transition state using a non-hydrolyzable dipeptide isostere at the cleavage site. It was found that the double mutant APP with Asn670-Leu671Asp672 (Swedish APP) is a more efficient substrate for BACE1 cleavage than wild-type APP (Lys670Met671-Asp672). Also, SAR studies demonstrated that $\mathrm{P} 1$ ' position shows high preference for alanine residue. Consequently, the design of the substratebased inhibitor compound included the involvement of non-hydrolysable Leu-Ala hydroxyethylene dipeptide isostere at scissile site.

OM99-2 inhibitor exhibits potent BACE1 inhibitory activity with Ki of $1.6 \mathrm{nM}$ (A. K. Ghosh et al. 2000). It binds to BACE1 in substrate binding pocket between $\mathrm{N}$ and $\mathrm{C}$-lobes of enzyme in an extended conformation. While Asp32 and Asp228, the catalytic dyad are located in the center of binding pocket and interact with the hydroxyl group of the transition-state isostere through a network of hydrogen bonds. 


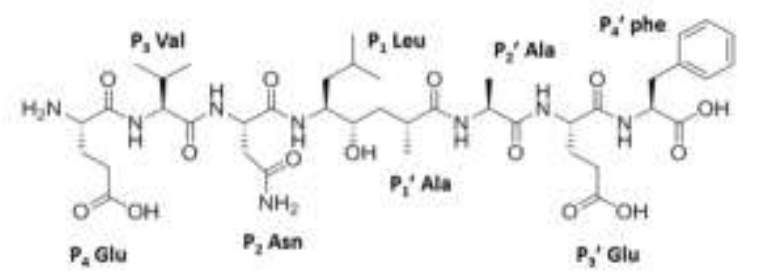

Fig.(4): Structure of OM99-2 inhibitors with its eight residues

Depending on the previous molecular insight, Ghosh and Tang were able to develop OM00-3 inhibitor (inhibitor 2) by further optimization of their octapeptide inhibitor structure (OM99-2) (Turner et al. 2001). OM00-3 (Fig. 5) showed a Ki value of $0.3 \mathrm{nM}$ (Hong et al. 2002), about five folds more potent than OM99-2 against BACE1. It was found that backbone conformation of OM00-3 from P3 to P2' was retained as in complex of OM99-2 and BACE1 (Hong et al. 2002). Unlike OM99-2, OM00-3 backbone at $\mathrm{P}^{\prime}$ and $\mathrm{P} 4{ }^{\prime}$ displayed an extended conformation that was stabilized by a hydrogen bond between carbonyl of P3' residue with $\operatorname{Arg} 128$ and a weak hydrogen bond from nitrogen of backbone P4' to Try198.

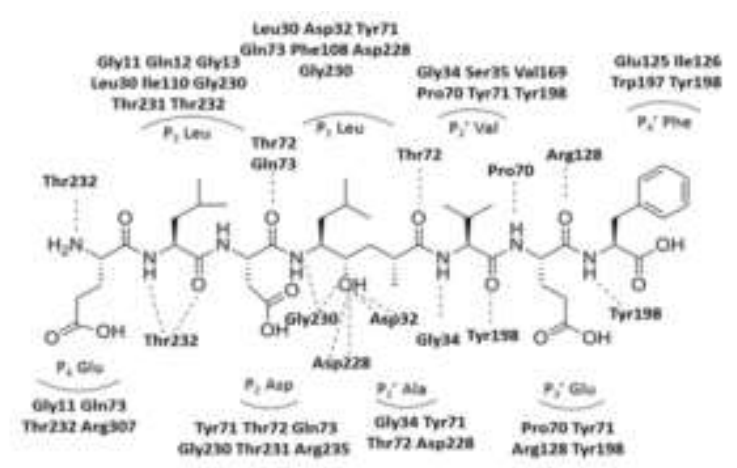

Fig.(5): Structure of OM00-3 inhibitor showing its interaction with $B A C E 1$ residues in the active site where the hydrogen bonds are represented with dached lines.

Whereas the druggability of these substrate-based inhibitors is restricted due to their high molecular weight, their provided molecular insight represents the initial and critical step in evolution and development of diversity of structure-based BACE1 inhibitors.

Many efforts have been devoted by pharmaceutical industries and academia for development of small size, potent, selective and biologically active BACE1 inhibitors. The developed inhibitors can be divided to 2 main classes: peptidomimetic and nonpeptidic inhibitors where further classification can be carried out based on their chemical structure.

\subsection{Structure-based BACE1 inhibitors 3.2.1 Design of peptidomimetic inhibitors}

Peptidomimetic inhibitors are developed by simulation of the natural substrate of BACE1 enzyme. Therefore, hydrogen bonding and hydrophobic interaction are permitted to be conserved or promoted by further side chains optimization. Peptidomimetic inhibitors can be assorted based on their transition state isostere.

\subsubsection{Statine -and norstatine-based inhibitors:}

Statines are used for development of transition state inhibitors because of presence of P1 leucine side chain simulating the Swedish double mutant APP. Statine based inhibitors has been broadly studied. Compound 3 was developed as statine based inhibitors and exhibited $110 \mu \mathrm{m}$ BACE1 inhibitory activity (J. Hu et al. 2003). Docking studies revealed that $\mathrm{S} 2$ and $\mathrm{S} 3$ pockets accommodate $\mathrm{P} 2$ alanine and P3 isoleucine residues respectively while isobutyl $\mathrm{P} 1$ group is located in $\mathrm{S} 1$ subsite.

Phenylstatine-based inhibitors are incorporated to introduce a larger hydrophobic substituent at P1 position as in compound 4 that showed a BACE1 $\mathrm{IC}_{50}$ of $21 \mathrm{nM}$ (20 folds more potent than inhibitors with 2-methylbutyl group) (B. Hu et al. 2004). Molecular modeling of compound 4 in BACE1 active site demonstrated that phenylstatine isostere formed hydrogen bonds with the catalytic aspartic dyad Asp32 and Asp228 of BACE1 and P1 benzyl occupied finely $\mathrm{S} 1$ pocket. Also, kimura et al. have reported compound 5 as phenylnorstatine based inhibitors (transition state isostere) that displayed BACE1 IC50 of $4.7 \mathrm{nM}$ (Kimura et al. 2004). Due to instability of this compound, they developed compound $6\left(\mathrm{IC}_{50}=3.9 \mathrm{nM}\right)$ by incorporation of $1 \mathrm{H}-$ tetrazole-5-carbonyl substituent at $\mathrm{P} 4$ position instead of oxalyl moiety as bioisostere (Kimura et al. 2005). Modeling studies showed that the introduced P4 tetrazole ring forms hydrogen bonds with Arg235 and Arg307 residues. Although the above compounds exhibit potent BACE1 inhibitory activity, the prescence of numerous polar moieties prevents their penetration across cell membrane and BBB. Therefore, further optimization involves introduction of hetrocyclic $1 \mathrm{H}$-tetrazole ring at position $1^{\prime}$ as a suitable bioisostere of carboxylic acid which resulted in compound 7 with potent BACE1 IC50 of $1.2 \mathrm{nM}$ (Kimura et al. 2006). 


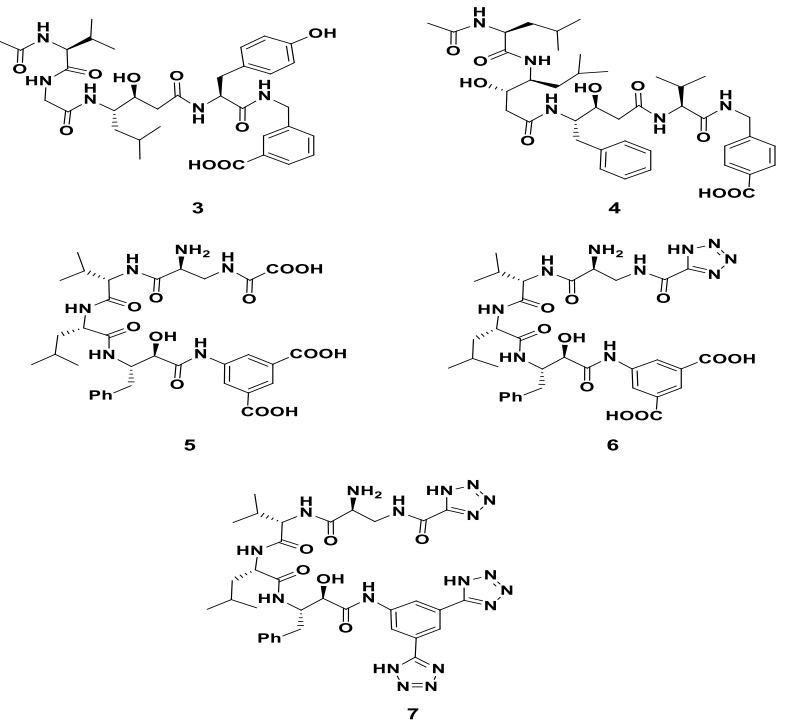

3.2.1.2 Hydroxyethylene-based inhibitors

Hydroxyethylene based inhibitors were amongst the first developed BACE1 inhibitors. The first designed BACE1 inhibitors, OM99-2 and OM00-3 are based on using hydroxyethylene core as transition state isostere. Hydroxyethylene based inhibitor 8 was designed by cutting off $\mathrm{P}^{\prime}{ }^{\prime}, \mathrm{P} 4{ }^{\prime}$ and P4 sites from OM99-2 inhibitor. This compound showed a Ki value of $2.5 \mathrm{nM}$ (A. K. Ghosh et al. 2001). Different ligands such as methylcysteine and methyl-sulfone were investigated at $\mathrm{P} 2$ position but methionine, as demonstrated in compound 8, exhibited the most potent activity due to its interaction with $\operatorname{Arg} 235$ residue (A. Ghosh et al. 2006). However, this inhibitor was non-selective for BACE1 enzyme. In an attempt for development of selective BACE1 inhibitors, inhibitor 9 was designed with introduction of pyrazolylmethyl urethate at position $\mathrm{P} 3$. This inhibitor was selective for BACE1 enzyme and displayed a $K_{i}$ value of 0.3 nM (A. K. Ghosh et al. 2006). Docking studies revealed that one of pyrazole ring nitrogen's forms hydrogen bonds with Thr232 and one of methyl substituents on pyrazole ring locates into hydrophobic S3 subsite. While P2 sulfone moiety forms hydrogen bonds with Arg235 residue and a tightly bound water molecule. It was suggested that these interactions may be responsible for the selectivity of inhibitor 9. Further optimization resulted in inhibitor 10 with an oxazolylmethyl moiety at position P3. Inhibitor 10 showed a $K_{i}$ of $0.12 \mathrm{nM}$ and was highly selective for BACE1 over BACE2 and cathepsin D (A. K. Ghosh et al. 2006). Although hydroxyethylene-based inhibitors are potent, optimization of their structures are required to minimize their molecular size and enhance their drug-like properties (Tang, Hong, and Ghosh 2010).
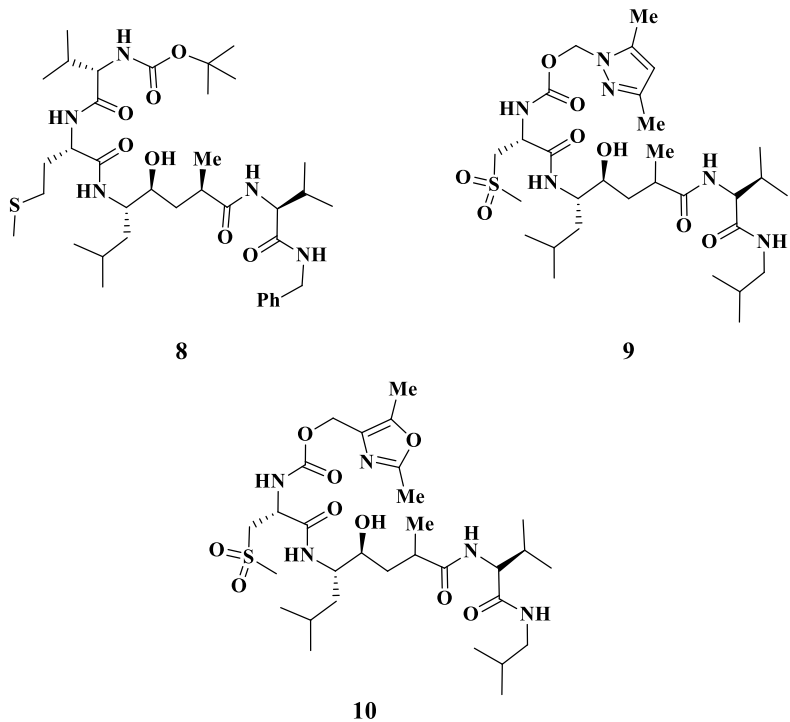

\subsubsection{Hydroxyethylamine-based inhibitors}

Utilizing the information and molecular insight gained from the previously discussed inhibitors, pepeptidomimetic inhibitors including hydroxyethylamine isosteres have been developed (Stachel et al. 2004). Hydroxylethylamine inhibitor 11 displayed an enzyme $\mathrm{IC}_{50}$ of $15 \mathrm{nM}$ and a cellular $\mathrm{IC}_{50}$ of $29 \mathrm{nM}$. An X-ray co-crystal structure of inhibitor 11 complexed with BACE1 enzyme demonstrated that hydroxyethyl amine moiety binds with catalytic asparatic dyad where hydroxyl group forms hydrogen bonds with Asp32 residue and $\alpha$ amino group binds with Asp228. Also, P3 $\alpha-$ methylbenzylamide fits into S3 pocket and P2 sulfonamide oxygen hydrogen bonds to backbone $\mathrm{NH}$ of Thr232 and Asn233 residues.

Further investigation efforts resulted in design of inhibitor 12 with introduction of benzamide moiety on the non-prime side in order to improve metabolic stability and cellular uptake of preceding inhibitors (Clarke et al. 2008). Docking studies showed that carbonyl group of lactam ring was involved in a hydrogen bond with Asn294 while the nitrogen linked alkyl chains fills in S3 pocket. It was highly selective for BACE1 over BACE2 and cathepsin D with IC50 of $13 \mathrm{nM}$ (Clarke et al. 2008).

Inhibitor 13 was developed by combining hydroxyethylamine moiety with isophthalamide moiety at position 2 and 3-methoxybenzene moiety at $\mathrm{P} 2^{\prime}$ position. It showed very potent inhibitory activity with $K_{\mathrm{i}}$ value of $1.8 \mathrm{nM}$ and selectivity over BACE2 and cathepsin D (A. K. Ghosh et al. 2008). Also, conformationally constrained hydroxyethylamine inhinitors have been developed. The design of these inhibitors was based on incorporation of conformationally constrained cyclic amines to bind with catalytic aspartic dyad 
and fill in $\mathrm{S}^{\prime}$ site. Inhibitor 14 is a representative example that exhibited an IC50 of $71 \mathrm{nM}$ (Barrow et al. 2007). Modeling studies revealed that imidazolidinone carbonyl has contacts in flap region and the prontonated nitrogen hydrogen bonds to Asp228 and Gly34. Further optimization to improve cellular potency, inhibitor 15 was designed with a pyrazole moiety as the constraining hydroxyethylamine isosteres ( $\mathrm{Zou}, \mathrm{Xu}$, et al. 2013). It was found that inhibitor 15 demonstrated superior membrane permeability with an enzyme IC50 of $230 \mathrm{nM}$.

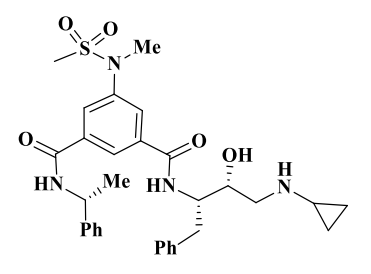

11

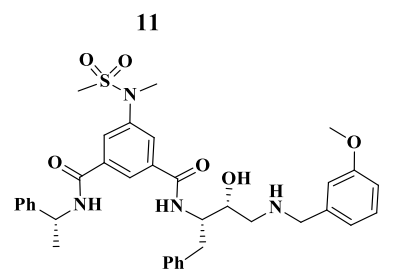

13

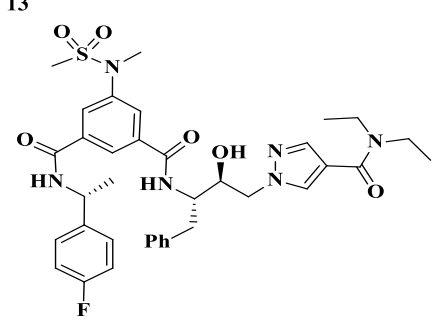

15

\subsubsection{Carbinamine-derived inhibitors}

In an attempt to enhance the penetration of blood brain barrier while preserving the accomplished potency in previously designed inhibitors, carbinamines-derived inhibitors have been investigated. Inhibitor 16 was developed with incorporation of 2, 6 diamino-isonicotinamide moiety as the aspartate binding element. It showed a cellular IC50 value of $49 \mathrm{nM}$ and in vivo activity in transgenic mice (Stauffer et al. 2007). A combination of isonicotinic moiety including methylcyclopropl group at P3 position with oxadiazolyl tertiary carbinamine led to development of inhibitor 17. The crystal structure of 17 complexed with BACE1 demonstrated that inhibitor 17 fit into S1 and S3 pockets where the benzyl moiety occupied S1 sites. Although inhibitor 17 showed a very potent inhibitory activity with IC50 value of $0.4 \mathrm{nM}$ with good potential for brain penetration, it exhibited unfavorable pharmacokinetic properties due to poor oral bioavailability (Nantermet et al. 2009).

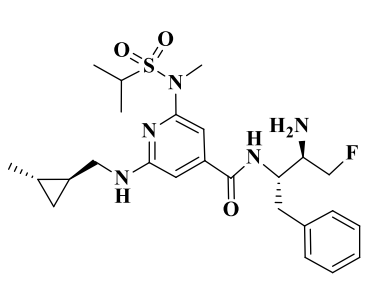

16

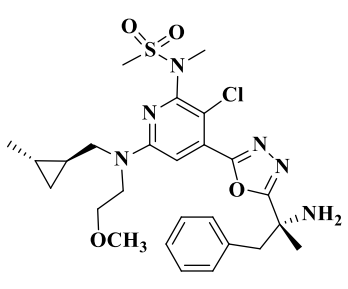

17

\subsubsection{Design of non- peptidic inhibitors}

Due to the large molecular size, law oral bioavailability, metabolic instability and poor blood brain barrier penteration (BBB) of peptidomimetic inhibitors, many researches have focused on evolution of small molecules and non-peptidic inhibitors. A high throughput screening has been conducted followed by chemical optimization that resulted in identification of diversity of nonpeptidic scaffolds for design of BACE1 inhibitors. The major goals for research are development of non-peptidic BACE1 inhibitors that are smaller in size with enhanced $\mathrm{BBB}$ penetration and drug like properties. The development in the structure based design of non peptidic inhibitors among various scaffolds will be discussed below:

\subsubsection{2- aminopyridine based-inhibitors}

2-aminopyridine scaffold has been involved in development of small molecules non-peptidic inhibitors. BACE1 enzyme adapts open flap conformation upon bounding to 2-aminopyridine inhibitors where Try71 residue was located above the pyridine moiety. Following various in silico screening studies, 2,6 -substituted aminopyridine hit 18 was identified with reasonable an $\mathrm{IC}_{50}$ of $25 \mu \mathrm{m}$ (Congreve et al. 2007).

Further optimization resulted in inhibitor 19 where indole moiety was incorporated to enhance cellular potency of inhibitors. It exhibits an $\mathrm{IC}_{50}$ of $690 \mathrm{nM}$ while modeling studies revealed that indole nitrogen interacts with Gly230 and 2,3 diamino pyridine moiety is laid under the flap. Inhibitor 20 was developd with introduction of pyrole moiety where it demonstrated an enhanced cellular potency $\left(\mathrm{EC}_{50}=440 \mathrm{nM}\right)($ Malamas, Barnes, et al. 2010). Its binding mode showed that the aminopyridine moiety form hydrogen bonds to catalytic aspartic acids Asp32 and Asp228 and pyrimidine ring hydrogen bonds to Ser229 while pyrole 
functionality makes a $\Pi$ stacking interaction with Try71.<smiles>COc1cccc(-c2cccc(CCc3cccc(N)n3)c2)c1</smiles>

18

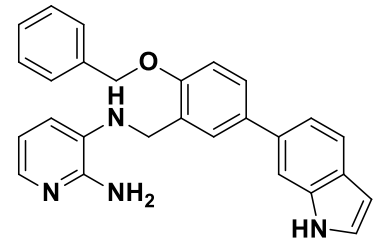

19

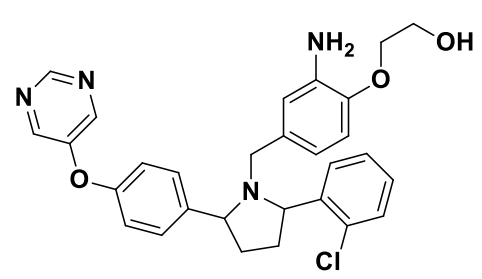

20

\subsubsection{Aminoimidazole-based inhibitors}

Utilizing aminoimidazole heterocyclic scaffold, potent small molecule BACE1 inhibitors have been promoted. High throughput screening resulted in initial hit 21 that displayed weak enzyme inhibitory potency. Next modification led to development of conformationally constrained inhibitor 22 which exhibited a viable ability for BBB penetration with an enzyme $\mathrm{IC}_{50}$ value of $63 \mathrm{nM}$ (Hills et al. 2009). It was suggested that increase in activity may be due to increasing hydrophobic interaction in BACE1 flap region through the introduced indane moiety. Further investigation yielded fused aminoimidazole inhibitors in an attempt to improve brain penetration and potency. Inhibitor 23 was developed as the initial hit by combination of bicyclic aminoimidazole functionality with a biphenyl moeity where it exhibited $\mathrm{IC}_{50}$ of $38 \mu \mathrm{M}$ (Malamas et al. 2009). Subsequent optimization resulted in inhibitor 24 with p-difloromethoxy substituent on one phenyl groups and an m-alkynyl substituent on the other group. Inhibitor 24 demonstrated a potent enzymatic inhibitory activity with $\mathrm{pIC}_{50}$ of 7.11 and also showed a good potency in cellular assay with $\mathrm{pIC}_{50}$ value of 7.46 (Swahn et al. 2012). Its binding mode showed that aminoimidazole

moiety hydrogen bonds to catalytic aspartic acids Asp32 and Asp228 in an open flap conformation. Also, oxygen of p-difloromethoxy substituent forms a hydrogen bond with Try 76 residue while m-alkynl substituent fits into S3 hydrophobic subsite.
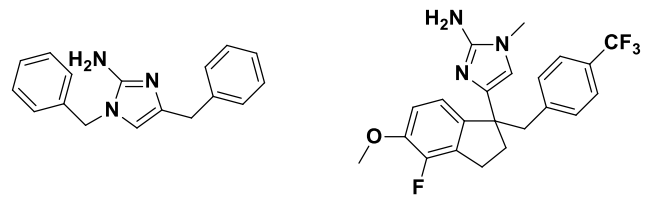

21

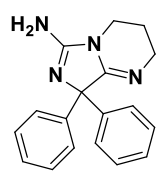

23

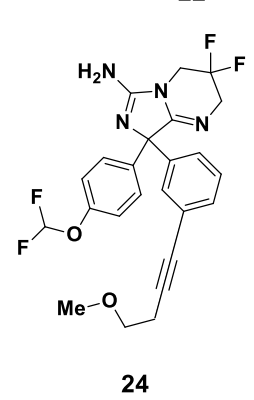

\subsubsection{Aminohydantoin/- Iminohydantoin-} based Inhibitors

High throughput screening identified hit 25 with an aminohydantion scaffold, which showed an enzymatic IC50 value of $3.4 \mu \mathrm{M}$ (Malamas, Erdei, et al. 2010). This led to subsequent development of potent aminohydantion based inhibitors. Inhibitor 26 was developed in an attempt to optimize hydrophobic functionality and therefore improve the potency. It showed good inhibitory activity with an IC50 of $10 \mathrm{nM}$ and good cellular potency with EC50 of $20 \mathrm{nM}$ (Malamas, Erdei, et al. 2010). Modeling studies illustrated that amino group and pharmacophore N-H involves in hydrogen bonding interactions with catalytic aspartic dyad while pmethoxy benzyl group interacts with Try76 in S2' pocket. Moreover, phenyl pyridine moiety occupies hydrophobic S3 pocket interacting with Ser229 through a bridge of water molecules. Inhibitor 26 exhibited good selectivity for BACE1 against BACE2, cathepsin D and other aspartic proteases. In vivo studies in mouse models manifested that plasma A $\beta 40$ was reduced by $69 \%$ after a 100 $\mathrm{mg} / \mathrm{kg}$ oral administration of inhibitor 26 (Malamas, Erdei, et al. 2010).

Inhibitor 27 was developed using iminohydantion scaffold, showed good BACE1 activity with IC50 value of $22 \mathrm{~nm}$ (Caldwell et al. 2014). It binding mode showed that quinolone moiety locates in solvent exposed region and involves in a hydrogen bonding interaction with Phe108 carbonyl. Furthermore, it also has hydrophobic interactions with Lys107 and Ile110 residues. Unfortunately, inhibitor 27 wasn't able to reduce brain $A \beta 40$ after dosed in a mouse model although it cleared plasma $\mathrm{A} \beta 40$ by $65 \%$ and $55 \%$ after oral and subcutaneous doses in rats. 


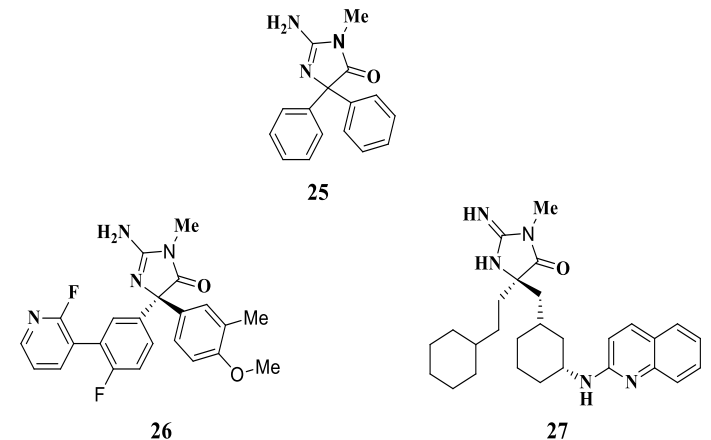

\subsubsection{Aminothiazoline- and Aminooxazoline- Based inhibitors}

Aminothiazoline based inhibitors evolved from the initial hit 28 which showing a BACE1 IC50 of 41.2 $\mu \mathrm{M}$ (Woltering et al. 2013). Modeling studies elucidated that meta-substitution on phenyl group enhance the hydrophobic interaction in S3 pocket. Utilizing SAR studies, inhibitor 29 was developed showing good inhibitory activity with IC50 of 27 $\mathrm{nM}$ and cellular $\mathrm{A} \beta$ inhibitory activity with IC50 of $2 \mathrm{nM}$ (Woltering et al. 2013). It was found that meta-substituted amide moiety permits phenyl group to hydrogen bonds to Gly291 residue and therefore adapting almost planar conformation. Further investigation yielded aminooxazoline based inhibitors. Inhibitor 30 was developed with introduction of a triflorometyl substituent on oxazoline hetrocyclic ring. It displayed a BACE1 IC50 of $12 \mathrm{nM}$ and cellular $A \beta$ inhibitory activity of $2 \mathrm{nM}$ (Hilpert et al. 2013). Moreover, inhibitor 30 exhibited good selectivity over other aspartic proteases and reduced $A \beta 40 / 42$ level after oral administration showing promising pharmacokinetic properties.
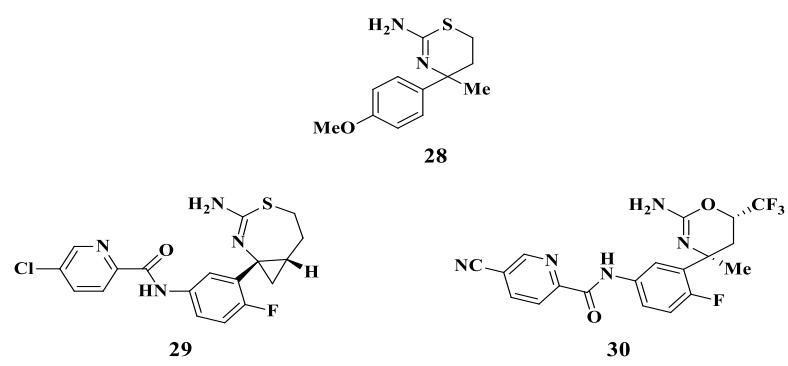

\subsubsection{Dihydroquinazoline-based inhibitors}

Identification of fragment hit 31 resulted in design of BACE1 inhibitors incorporating dihydroquinazoline scaffold (Baxter et al. 2007). Xray crystal structure of 31 in BACE1 active site demonstrated that $\mathrm{N}$-cyclohexyl group occupies $\mathrm{S} 1$ pocket while $\mathrm{S1}^{\prime}$ subsite was remained unoccupied.
Consequently, inhibitor 32 was developed with introduction of a cyclohexyl moiety to fill S1' pocket and therefore improving ligand binding (Baxter et al. 2007). Although, it exhibited enzymatic $\mathrm{Ki}$ value of $11 \mathrm{nM}$ and showed reasonable selectivity over other aspartic proteases, it showed a poor brain penetration. Subsequent optimization resulted in inhibitor 33 that incorporates methoxy methyl thiazole moiety in an attempt to improve binding interaction in the active site (A. K. Ghosh et al. 2012). Molecular modeling of inhibitor revealed that methyl ethyl oxygen forms a hydrogen bonding interaction with Thr232 residue. It displayed better enzymatic inhibitory activity (IC50 = $13 \mathrm{nM}$ ) and cellular inhibitory activity (IC50 $=21 \mathrm{nM})$.

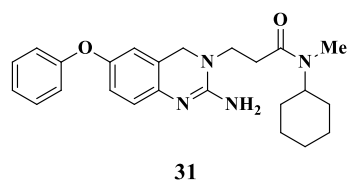

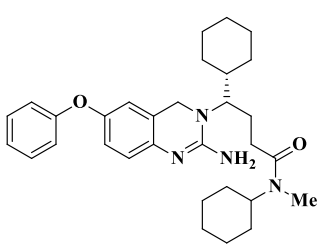

32

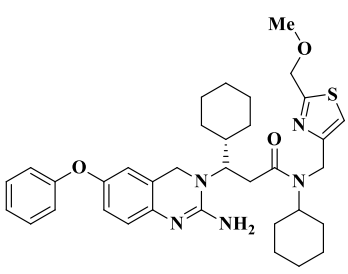

33

\subsubsection{Aminoquinoline-based inhibitors}

Fragment based screening produced aminoquinoline derivative 34 exhibiting BACE Kd of $900 \mu \mathrm{M}$ (Cheng et al. 2011). Extensive SAR studies revealed that aromatic substitution on aminoquinoline ring at position 6 exhibited superior potency than substitution at any other position. Moreover, ortho- or meta- substitution on the ring may improve potency while para-substitution wasn't appropriate in the active site. Further structural modification suggested that introduction of branched alkyl amide at position 3 could extend into the S2' pocket.

Inhibitor 35 exhibited good BACE1 activity (IC50 $=74 \mathrm{nM})$. The introduced N-cyclohexyl propionamide at $\mathrm{C} 3$ of aminoquinoline ring was oriented toward $\mathrm{S} 2$ ' site while methyl of O-tolyl moiety at position 6 was located in P1 site (Cheng et al. 2011).

In order to improve pharmacokinetic properties, inhibitor 36 was designed with chloropyridyl moiety at position 6 (Cheng et al. 2011). It exhibited better BACE1 IC50 of $11 \mathrm{nM}$ and cellular IC50 of $80 \mathrm{nM}$. In vivo studies revealed that after subcutaneous administration of $60 \mathrm{mg} / \mathrm{Kg}$ of inhibitor 36 in rats, reduction of cerebrospinal fluid 
A $\beta$ levels by $42 \%$ was observed. But metabolic stability of inhibitor 38 wasn't satisfied due to its rapid clearance in human and rat liver microsomes. Modeling studies showed that quinolone ring involved in a strict interaction with Tyr71 residue, which direct it and nitrogen of amino group toward catalytic aspartic dyad Asp228 and Asp32 forming hydrogen bonds interactions.

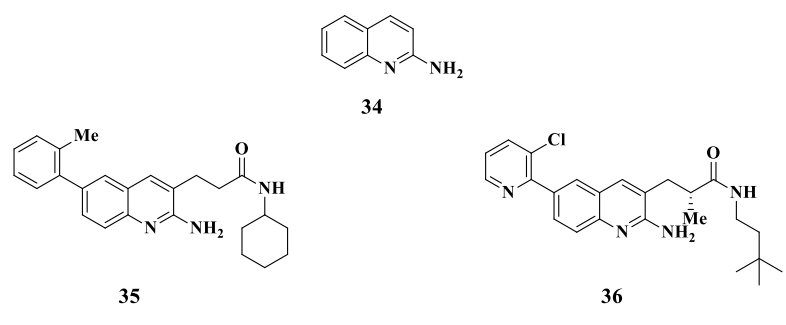

\subsubsection{Pyrrolidine-based inhibitors}

High throughput screening of a library of compounds resulted in identification of BACE1 inhibitors withwith pyrolidine scaffold. Initial hit 37 displayed an IC50 value of $240 \mu \mathrm{M}$ (Stachel et al. 2012). Subsequent structural modification resulted in inhibitor 38, exhibiting an enzymatic inhibitory acitivty of $29 \mathrm{nM}$. However, it wasn't selective for BACE1 against BACE2 (Stachel et al. 2012). Modeling studies revealed that pyrrollidine moiety was responsible for forming two hydrogen bonds with catalytic aspatrtic acid residues Asp228 and Asp32 while disubstituted piperdine functionality was located in a pocket under flap region.

\subsubsection{Acyl guanidine-based inhibitors}

Identification of BACE1 inhibitors containing acyl guanidine moiety was carried out using high

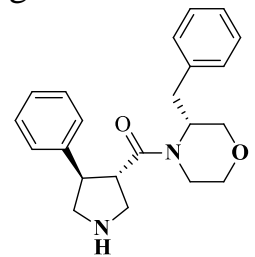

37

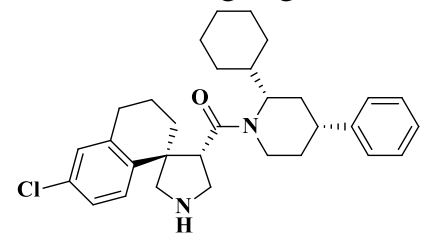

38 throughput screening. The initial hit 39 was developed showing BACE1 IC50 of $3.7 \mu \mathrm{M}$ (Cole et al. 2006). Binding mode of 40 demonstrated that acyl guanidine moiety forms four hydrogen bonds with catalytic dyad (Asp228 and Asp32) while biphenyl groups occupies both $\mathrm{S} 1$ and S2' pockets (Cole et al. 2006). Further optimization resulted in inhibitor 40, showing better enzymatic inhibitory activity $($ IC50 $=110 \mathrm{nM})$. Crystallographic studies of 40 in BACE1 active site revealed that acyl guanidine moiety makes hydrogen bonding interaction with catalytic aspartic acid residues while propyl alcohol substitution on one of the guanidine nitrogens accessed into S1' pocket forming hydrogen bonds with Arg235 and Thr329 residues. Furthermore, the p-propyloxyphenyl substituent extends across S1-S3 pockets. However, this compound exhibited low selectivity over other aspartic proteases.

A combination of an indole moiety with an acyl guanidine core yielded inhibitor 41, exhibiting BACE1 IC50 of $1.01 \mu \mathrm{M}$ (Zou, Li, et al. 2013). Modeling stuies demonstrated that acyl guanidine core hydrogen bonds to catalytic aspartic acid residues as mentioned before. Carbonyl group of acyl guanidine formed hydrogen bonds with Gln73 and Thr72 via water molecules. Moreover, the indole ring is responsible for a cation- $\Pi$ interaction with side chain of Arg235 residue in S1' subsite.

Subsequent optimization led to Inhibitor 42, with introduction of nitrile group on indole ring at position 6. It was found that this nitrile moiety formed a new hydrogen bond with Ser328 residue resulted in enhancing the enzymatic inhibitory activity with IC50 value of $44 \mathrm{nM}$ (Zou, Li, et al. 2013).<smiles>NC(N)=NC1Cc2c(N)cccc2-c2ccc(-c3ccccc3)n2C1</smiles>

39<smiles>CC(=O)Nc1c(Cl)cc(CNC(N)=NC(=O)Cn2ccc3ccccc32)cc1Cl</smiles>

41<smiles>CCCOc1ccc(-c2ccc(-c3ccccc3Cl)n2CC(=O)/N=C(/N)NCCCO)cc1</smiles>

40<smiles>CC(=O)Nc1c(Cl)cc(CNC(N)=NC(=O)Cn2ccc3ccc(C#N)cc32)cc1Cl</smiles>

42

\subsubsection{Beta secretase inhibitors in clinical trials}

Until now no BACE1 inhibitors have been FDA approved for treatment of $\mathrm{AD}$ however, there are numbers of candidates that have reached various stages of clinical trials. EliLilly evolved Inhibitor 45 (LY2811376) which based on aminothiazine scaffold (Uddin et al. 2020). It exhibited BACE1 $\mathrm{IC}_{50}$ of $240 \mathrm{nM}$ (A. K. Ghosh and Osswald 2014). During with phase I clinical trial which started in 2009 , toxicology studies on rats observed presence of retinal pathology in these animals. Therefore, the ongoing clinical investigations of LY811376 were discontinued (Uddin et al. 2020). 
Inhibitor 46 (LY-2886721) was developed and studied clinically by EliLilly. It was also based on aminothiazine scaffold where it represented the first BACE1 inhibitor advanced to phase II clinical trials. It showed satisfactory results in phase I clinical trials and subsequently advanced to phase II trials in 2012 which carried out on patients with mild AD. During the study, routine monitoring observed marked increase in liver enzymes levels in 4 of out 70 patients. Therefore, phase II trial of LY-2886721 was discontinued (Uddin et al. 2020).

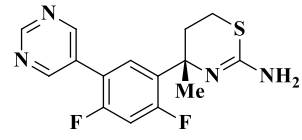

Inhibitor 45 (LY-2811376)

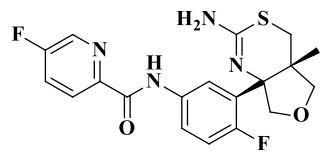

Inhibitor 46 (LY-2886721)
Merck developed inhibitor 47 (verubecestat or MK8931) using iminopyrimidinone scaffold (Uddin et al. 2020). It showed adequate pharmacokinetic properties in animal models and caused marked decrease in CSF A 340 levels in monkeys by $81 \%$ at dose $10 \mathrm{mg} / \mathrm{kg}$. Verubecestat reached phase II and phase III trials in 2012 that carried out on patients with mild to moderate AD. But unfortunately, in feburary 2017, Merck research laboratories have declared discontinue of clinical trials on MK-8931 due to poor efficacy and existence of synaptic and neuronal damage (Uddin et al. 2020).

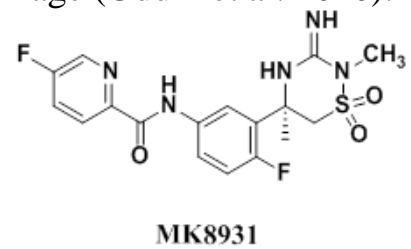

Astrazenica evolved inhibitor 48 (AZD-3239) that based on aminoimidazole scaffold where it reached phase I trial in 2012 (Uddin et al. 2020). The clinical studies demonstrated that single dose of AZD-3239 reduces the mean plasma concentration of $\mathrm{A} \beta 42$ and $\mathrm{A} \beta 40$ by $70 \%$ while prolonged inhibition was obtained by only one week dose regimen. Recently, AZD-3239 advances to phase III clinical trial where Eli Lilly and AstraZeneca are sponsoring these trials which carried out on 4000 patients with mild AD in order to estimate the potency of AZD-3239 (Uddin et al. 2020).

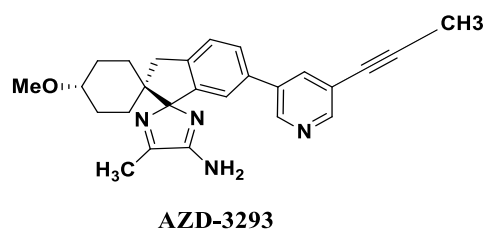

\section{Conclusion}

Alzheimer's disease is the most prevalent neurodegenerative disease that represents about 60 $80 \%$ of total cases of dementia all over the world. FDA approved treatment for AD relieves symptoms only without effect on progression of neurodegeneration process in AD. Therefore, many efforts have been dedicated to discover novel disease modifying therapies can retard or halt the progression of disease.

Beta secretase is a promising target for evolution of inhibitors that can inhibit the progression of disease. Crystal structure of $\beta$-secretase enzyme has been determined either alone or in a complex with small inhibitors and that permitted the design of structure based BACE1 inhibitors. Since its discovery, there are many endeavors have been devoted for development of small molecule and potent BACE1 inhibitors. Starting from substrate based inhibitors, peptidomimetic inhibitors and recently non peptidic inhibitors which showing much more affinity for BACE1. Many $\beta$-secretase inhibitors have emerged and some of which have already reached the different stages of clinical trials.

\section{References}

"2020 Alzheimer's Disease Facts and Figures." 2020. Alzheimer's \& Dementia 16(3): 391-460. https://alz-

journals.onlinelibrary.wiley.com/doi/abs/10.1002/a lz.12068.

Ali, Gemma-Claire et al. 2015. "Chapter 2: The Global Prevalence of Dementia." World Alzheimer Report.

Barrow, Jamesemsp14C et al. 2007. "Design and Synthesis of 2, 3, 5-Substituted Imidazolidin-4-One Inhibitors of BACE-1." ChemMedChem 2(7): 99599.

Baxter, Ellen W et al. 2007. "2-Amino-3, 4Dihydroquinazolines as Inhibitors of BACE-1 ( $\beta$ Site APP Cleaving Enzyme): Use of Structure Based Design to Convert a Micromolar Hit into a Nanomolar Lead." Journal of medicinal chemistry 50(18): 4261-64.

C Vickers, James et al. 2016. "Defining the Earliest Pathological Changes of Alzheimer's Disease." Current Alzheimer Research 13(3): 281-87.

Caldwell, John P et al. 2014. "Discovery of Potent Iminoheterocycle BACE1 Inhibitors." Bioorganic \& medicinal chemistry letters 24(23): 5455-59.

Cheng, Yuan et al. 2011. "From Fragment Screening to in Vivo Efficacy: Optimization of a Series of 2-Aminoquinolines as Potent Inhibitors of Beta-Site Amyloid Precursor Protein Cleaving Enzyme 1 (BACE1)." Journal of medicinal chemistry 54(16): 5836-57. 
Clarke, Brian et al. 2008. "BACE-1 Inhibitors Part 1: Identification of Novel Hydroxy Ethylamines (HEAs)." Bioorganic \& medicinal chemistry letters 18(3): 1011-16.

Coates, Leighton et al. 2006. "X-Ray, Neutron and NMR Studies of the Catalytic Mechanism of Aspartic Proteinases." European Biophysics Journal 35(7): 559-66.

Cole, Derek C et al. 2006. "Acylguanidines as Small-Molecule \$ \$-Secretase Inhibitors." Journal of medicinal chemistry 49(21): 6158-61.

Congreve, Miles et al. 2007. "Application of Fragment Screening by X-Ray Crystallography to the Discovery of Aminopyridines as Inhibitors of $\beta$ Secretase." Journal of medicinal chemistry 50(6): 1124-32.

Femminella, Grazia Daniela et al. 2018. "Imaging and Molecular Mechanisms of Alzheimer's Disease: A Review." International Journal of Molecular Sciences 19(12): 3702.

Ghosh, Arun et al. 2006. "Beta-Secretase Inhibitors and Methods of Use. U.S. Patent Application No 10/493,439.

Ghosh, Arun K et al. 2000. "Design of Potent Inhibitors for Human Brain Memapsin 2 ( $\beta$ Secretase)." Journal of the American Chemical Society 122(14): 3522-23.

Ghosh, Arun K et al. 2001. "Structure-Based Design: Potent Inhibitors of Human Brain Memapsin 2 (\$\$\$-Secretase)." Journal of medicinal chemistry 44(18): 2865-68.

Ghosh, Arun K et al. 2006. "Design, Synthesis and $\mathrm{X}$-Ray Structure of Protein- Ligand Complexes: Important Insight into Selectivity of Memapsin 2 ( $\beta$ Secretase) Inhibitors." Journal of the American Chemical Society 128(16): 5310-11.

Ghosh, Arun K., et al. 2008. "Potent Memapsin 2 ( $\beta$-Secretase) Inhibitors: Design, Synthesis, ProteinLigand X-Ray Structure, and in Vivo Evaluation." Bioorganic \& medicinal chemistry letters 18(3): 1031-36.

Ghosh, Arun K., et al. 2012. "Structure-Based Design, Synthesis, and Biological Evaluation of Dihydroquinazoline-Derived Potent $\beta$-Secretase Inhibitors." Bioorganic \& medicinal chemistry letters 22(17): 5460-65.

Ghosh, Arun K, and Heather L Osswald. 2014. "BACE1 ( $\beta$-Secretase) Inhibitors for the Treatment of Alzheimer's Disease." Chemical Society Reviews 43(19): 6765-6813.

Halima, Saoussen Ben et al. 2016. "Specific Inhibition of $\beta$-Secretase Processing of the Alzheimer Disease Amyloid Precursor Protein." Cell Reports 14(9): 2127-41.
Harrison, Timothy, and Dirk Beher. 2003. " $\gamma-$ Secretase Inhibitors--from Molecular Probes to New Therapeutics?" Progress in medicinal chemistry 41: 99-127.

Hills, Ivory D et al. 2009. "A Conformational Constraint Improves a $\beta$-Secretase Inhibitor but for an Unexpected Reason." Bioorganic \& medicinal chemistry letters 19(17): 4993-95.

Hilpert, Hans et al. 2013. " $\beta$-Secretase (BACE1) Inhibitors with High in Vivo Efficacy Suitable for Clinical Evaluation in Alzheimer s Disease." Journal of medicinal chemistry 56(10): 3980-95.

Hong, Lin et al. 2000. "Structure of the Protease Domain of Memapsin 2 ( $\$ \beta \$$-Secretase) Complexed with Inhibitor." Science 290(5489): 150-53.

Hong, Lin, et al. 2002. "Crystal Structure of Memapsin 2 (\$ $\beta \$-$ Secretase) in Complex with an Inhibitor OM00-3." Biochemistry 41(36): 1096367.

Hong, Lin, and Jordan Tang. 2004. "Flap Position of Free Memapsin 2 (\$\$\$-Secretase), a Model for Flap Opening in Aspartic Protease Catalysis." Biochemistry 43(16): 4689-95.

Hu, Baihua et al. 2004. "Synthesis and SAR of BisStatine Based Peptides as BACE 1 Inhibitors." Bioorganic \& medicinal chemistry letters 14(13): 3457-60.

$\mathrm{Hu}$, Jingdan et al. 2003. "Design and Synthesis of Statine-Containing BACE Inhibitors." Bioorganic \& medicinal chemistry letters 13(24): 4335-39.

Kimura, Tooru et al. 2004. "KMI-358 and KMI370, Highly Potent and Small-Sized BACE1 Inhibitors Containing Phenylnorstatine." Bioorganic \& medicinal chemistry letters 14(6): 1527-31.

Kimura, Tooru et al. 2005. "Design and Synthesis of Highly Active Alzheimer's $\beta$-Secretase (BACE1) Inhibitors, KMI-420 and KMI-429, with Enhanced Chemical Stability." Bioorganic \& medicinal chemistry letters 15(1): 211-15.

Kimura, Tooru et al. 2006. "Design and Synthesis of Potent $\beta$-Secretase (BACE1) Inhibitors with P1' Carboxylic Acid Bioisosteres." Bioorganic \& medicinal chemistry letters 16(9): 2380-86.

Kontush, Anatol. 2004. "Apolipoprotein A $\beta$ : Black Sheep in a Good Family." Brain pathology 14(4): 433-47.

Korczyn, Amos D. 2008. "The Amyloid Cascade Hypothesis.” Alzheimer's \& dementia 4(3): 176-78. Lin, Xinli et al. 2000. "Human Aspartic Protease Memapsin 2 Cleaves the $\beta$-Secretase Site of $\beta$ Amyloid Precursor Protein." Proceedings of the National Academy of Sciences 97(4): 1456-60.

Malamas, Michael S et al. 2009. "Aminoimidazoles as Potent and Selective Human $\$ \beta \$$-Secretase 
(BACE1) Inhibitors." Journal of medicinal chemistry 52(20): 6314-23.

Malamas, Michael S, Jim Erdei, et al. 2010. "Design and Synthesis of 5, 5'-Disubstituted Aminohydantoins as Potent and Selective Human $\beta$ Secretase (BACE1) Inhibitors." Journal of medicinal chemistry 53(3): 1146-58.

Malamas, Michael S, Keith Barnes, et al. 2010. "Novel Pyrrolyl 2-Aminopyridines as Potent and Selective Human $\beta$-Secretase (BACE1) Inhibitors." Bioorganic \& Medicinal Chemistry Letters 20(7): 2068-73.

Nantermet, Philippe G et al. 2009. "Evolution of Tertiary Carbinamine BACE- 1 Inhibitors: $A \beta$ Reduction in Rhesus CSF upon Oral Dosing." ChemMedChem: Chemistry Enabling Drug Discovery 4(1): 37-40.

Nhan, Hoang S, Karen Chiang, and Edward H Koo. 2015. "The Multifaceted Nature of Amyloid Precursor Protein and Its Proteolytic Fragments: Friends and Foes." Acta neuropathologica 129(1): $1-19$.

Patterson, Christina. 2018. "The State of the Art of Dementia Research: New Frontiers." World Alzheimer Report. Reports, National Vital Statistics. 2018. "Deaths: Leading Causes for 2016."

Rhein, Virginie, and Anne Eckert. 2007. "Effects of Alzheimer's Amyloid-Beta and Tau Protein on Mitochondrial Function-Role of Glucose Metabolism and Insulin Signalling." Archives of physiology and biochemistry 113(3): 131-41.

Shimizu, Hideaki et al. 2008. "Crystal Structure of an Active Form of BACE1, an Enzyme Responsible for Amyloid $\beta$ Protein Production." Molecular and cellular biology 28(11): 3663-71.

Sinha, Sukanto et al. 1999. "Purification and Cloning of Amyloid Precursor Protein $\beta$-Secretase from Human Brain." Nature 402(6761): 537-40.

Stachel, Shawn J et al. 2004. "Structure-Based Design of Potent and Selective Cell-Permeable Inhibitors of Human $\beta$-Secretase (BACE-1)." Journal of medicinal chemistry 47(26): 6447-50.

Stachel, Shawn J., et al. 2012. "Discovery of Pyrrolidine-Based $\beta$-Secretase Inhibitors: Lead Advancement through Conformational Design for Maintenance of Ligand Binding Efficiency." Bioorganic \& medicinal chemistry letters 22(1): 240-44.

Stauffer, Shaun R et al. 2007. "Discovery and SAR of Isonicotinamide BACE-1 Inhibitors That Bind $\beta$ Secretase in a N-Terminal 10s-Loop down Conformation." Bioorganic \& medicinal chemistry letters 17(6): 1788-92.
Swahn, Britt-Marie et al. 2012. "Aminoimidazoles as BACE-1 Inhibitors: The Challenge to Achieve in Vivo Brain Efficacy." Bioorganic \& medicinal chemistry letters 22(5): 1854-59.

Tang, J, L Hong, and A K Ghosh. 2010. "Aspartic Acid Proteases as Therapeutic Targets." Wiley-VCH 45: 413-40.

Turner, Robert T et al. 2001. "Subsite Specificity of Memapsin 2 (\$\$\$-Secretase): Implications for Inhibitor Design." Biochemistry 40(34): 10001-6.

Uddin, Md. Sahab et al. 2020. "Novel AntiAlzheimer's Therapeutic Molecules Targeting Amyloid Precursor Protein Processing" ed. Fabiana Morroni. Oxidative Medicine and Cellular Longevity 2020: 7039138. https://doi.org/10.1155/2020/7039138.

Vassar, Robert et al. 1999. " $\beta$-Secretase Cleavage of Alzheimer's Amyloid Precursor Protein by the Transmembrane Aspartic Protease BACE." science 286(5440): 735-41.

Woltering, Thomas $\mathrm{J}$ et al. 2013. "BACE1 Inhibitors: A Head Group Scan on a Series of Amides." Bioorganic \& medicinal chemistry letters 23(14): 4239-43.

Yan, R et al. "Buhl. AE, Carter DB, Tomasselli AG, Parodi LA, Heinrikson RL, Gurney ME (1999) Membrane-Anchored Aspartyl Protease with Alzheimer's Disease Beta-Secretase Activity." Nature 402: 533-37.

Yuan, Jing et al. 2013. "Structure-Based Design of $\beta$-Site APP Cleaving Enzyme 1 (BACE1) Inhibitors for the Treatment of Alzheimer's Disease." Journal of medicinal chemistry 56(11): 4156-80.

Zou, Yiquan, Lei Xu, et al. 2013. "Discovery of Pyrazole as C-Terminus of Selective BACE1 Inhibitors." European journal of medicinal chemistry 68: 270-83.

Zou, Yiquan, Li Li, et al. 2013. "Virtual Screening and Structure-Based Discovery of Indole Acylguanidines as Potent $\beta$-Secretase (BACE1) Inhibitors." Molecules 18(5): 5706-22. 